\title{
Quantization of the Proca field in the Rindler wedge and the interaction of uniformly accelerated currents with massive vector bosons from the Unruh thermal bath.
}

\author{
Jorge Castiñeiras, ${ }^{*}$ Emerson B. S. Corrêa,$^{\dagger}$ and Luís C. B. Crispino ${ }^{\ddagger}$ \\ Faculdade de Física, Universidade Federal do Pará, 66075-110, Belém, Pará, Brazil \\ George E. A. Matsas ${ }^{\S}$ \\ Instituto de Física Teórica, Universidade Estadual Paulista, \\ Rua Dr. Bento Teobaldo Ferraz, 271-Bl. II, 01140-070 São Paulo, São Paulo, Brazil
}

(Dated: June 20, 2018)

\begin{abstract}
We canonically quantize the Proca field in the Rindler wedge and compute the total response rate of a uniformly accelerated current interacting with massive vector Rindler particles from the Unruh thermal bath. We explicitly verify that the result obtained is exactly the same as the emission rate of massive vector particles in the Minkowski vacuum as analyzed by inertial observers. Eventually our results are interpreted in terms of the interaction of static electrons coupled to $Z^{0}$ bosons present in Hawking radiation close to the event horizon of a black hole.

PACS numbers: 03.70.+k, 04.62.+v
\end{abstract}

\section{INTRODUCTION}

According to the Unruh effect [1] (see also Refs. [2, 3]), the vacuum state of a quantum field theory as described by inertial observers in Minkowski spacetime corresponds to a thermal state as seen by uniformly accelerated ones confined to the Rindler wedge (Rindler observers). By now, it is thoroughly accepted the essential role played by the Unruh effect in the description of various physical phenomena [4]. The analysis of the total response rate for classical and semiclassical sources with constant proper acceleration from the point of view of Rindler and inertial observers has been already performed for KleinGordon [5, 6], Maxwell [7] and Dirac fields [8].

Here we canonically quantize the Proca field (i.e., a massive spin-1 vector field) in the Rindler wedge and compute the response rate for a source with constant proper acceleration coupled to it. We explicitly verify that the total response rate as computed by Rindler observers immersed in the Unruh thermal bath is precisely the same as the one calculated by inertial observers in the Minkowski vacuum. Because uniformly accelerated sources are static in Rindler coordinates, they can only interact with zero-energy Rindler particles of the Unruh thermal bath. (We recall that this is possible because massive Rindler particles may have arbitrarily small frequencies as defined by uniformly accelerated observers [9].) Under proper conditions, our results can be interpreted in terms of noninertial electrons by saying that each $Z^{0}$ emitted from a uniformly accelerated $e^{-}$in the Minkowski vacuum as described by inertial observers corresponds to either the emission to or absorption from

\footnotetext{
*Electronic address: jcastin@ufpa.br

${ }^{\dagger}$ Electronic address: emerson@ufpa.br

‡Electronic address: crispino@ufpa.br

$\S$ Electronic address: matsas@ift.unesp.br
}

the Unruh thermal bath of a zero-energy $Z^{0}$ Rindler boson as described by coaccelerated observers.

The paper is organized as follows. In Sec. II, the Proca field is quantized in the Rindler wedge. In Sec. III, we briefly revisit the regularization procedure applied to static sources in the Rindler wedge as originally derived in Ref. [7]. In Sec. IV the corresponding emission and absorption rates of zero-energy Rindler particles are computed. In Sec. V it is explicitly verified that by combining the previously computed emission and absorption rates as calculated by Rindler observers, we exactly obtain the emission rate of (nonzero-energy) Minkowski particles as computed by inertial observers. In Sec. VI, we make our final remarks and comment on the response of a static charge interacting with $Z^{0}$ bosons of Hawking radiation in the vicinity of a black hole. We adopt natural units $\hbar=c=G=1$, unless stated otherwise.

\section{CANONICAL QUANTIZATION OF THE PROCA FIELD IN THE RINDLER WEDGE}

In order to quantize the Proca field we start with the standard Lagrangian density

$$
\mathcal{L}=\sqrt{-g}\left(-\frac{1}{4} F_{\mu \nu} F^{\mu \nu}+\frac{1}{2} m^{2} A_{\mu} A^{\mu}\right),
$$

where $g$ is the determinant of the metric $g_{\mu \nu}$ and $F^{\mu \nu} \equiv$ $\nabla^{\mu} A^{\nu}-\nabla^{\nu} A^{\mu}$. The field equations are

$$
\nabla_{\mu} F^{\mu \nu}+m^{2} A^{\nu}=0 .
$$

By applying $\nabla_{\nu}$ in Eq. (2), we obtain the Lorenz constraint

$$
\nabla_{\mu} A^{\mu}=0 .
$$

Equation (3) can be used to cast the field equation (2) in the form

$$
\left(\nabla^{\mu} \nabla_{\mu}+m^{2}\right) A^{\nu}=0,
$$


provided that the spacetime is a vacuum solution of Einstein equations: $R_{\mu \nu}=0$.

Now, let us proceed to quantize the Proca field in the (right) Rindler wedge, i.e., the portion of the Minkowski spacetime defined as $z>|t|$, which is a globally hyperbolic spacetime by its own right. Here $(t, \mathbf{x})$ with $\mathbf{x} \equiv(x, y, z)$ are the usual Cartesian coordinates. We cover the Rindler wedge using coordinates $(\tau, x, y, \xi)$, where the Rindler coordinates $\tau$ and $\xi$ are implicitly defined through

$$
t=\frac{e^{a \xi}}{a} \sinh a \tau, z=\frac{e^{a \xi}}{a} \cosh a \tau
$$

and $a=$ const $\in \mathbf{R}_{+}$. Rindler observers will be uniformly accelerated ones with constant coordinates $x, y, \xi$, and corresponding proper acceleration $a e^{-a \xi}$. By using Rindler coordinates, the metric components of the spacetime become

$$
g_{\mu \nu}=\operatorname{diag}\left(e^{2 a \xi},-1,-1,-e^{2 a \xi}\right) .
$$

In order to quantize the Proca field in the Rindler wedge, it is convenient to find a complete set of orthonormal solutions $A^{\left(\lambda, \varpi, \mathbf{k}_{\perp}\right)}$ for Eq. (4) satisfying the Lorenz constraint (3) associated with the three possible physical polarizations $\lambda=\mathrm{I}$, II, III:

$$
\left(A^{\left(\lambda, \varpi, \mathbf{k}_{\perp}\right)}, A^{\left(\lambda^{\prime}, \varpi^{\prime}, \mathbf{k}_{\perp}^{\prime}\right)}\right)=\delta_{\lambda \lambda^{\prime}} \delta\left(\varpi-\varpi^{\prime}\right) \delta^{2}\left(\mathbf{k}_{\perp}-\mathbf{k}_{\perp}^{\prime}\right),
$$

where $\mathbf{k}_{\perp} \equiv\left(k_{x}, k_{y}\right)$ represents the $z$-orthogonal momentum, and $\varpi \in(0,+\infty)$ labels the Rindler frequency. (We recall that $\varpi$ is distinct from the frequency $\omega \in(m,+\infty)$ of Minkowski modes.) For this purpose, we consider the generalized Klein-Gordon inner product

$$
\left(A^{(i)}, A^{(j)}\right)=\int_{\Sigma} d \Sigma n_{\mu} W^{\mu}\left(A^{(i)}, A^{(j)}\right)
$$

where $(i),(j)$ stand for the set of quantum numbers $\left(\lambda, \varpi, \mathbf{k}_{\perp}\right)$, the Cauchy surface $\Sigma$ will be chosen to be any $\tau=$ const hypersurface in the Rindler wedge, $n^{\mu} \propto$ $(1,0,0,0)$ is the future-pointing unit vector field orthogonal to $\Sigma$, and

$$
W^{\mu}\left(A^{(i)}, A^{(j)}\right) \equiv i\left(\overline{A_{\nu}^{(i)}} \pi^{(j) \mu \nu}-A^{(j)} \overline{\pi^{(i) \mu \nu}}\right)
$$

where the overline denotes complex conjugation, and

$$
\begin{aligned}
\pi^{(i) \mu \nu} & \equiv \frac{1}{\sqrt{-g}} \frac{\partial \mathcal{L}}{\partial\left(\nabla_{\mu} A_{\nu}^{(i)}\right)} \\
& =\nabla^{\nu} A^{(i) \mu}-\nabla^{\mu} A^{(i) \nu} \\
& =-F^{(i) \mu \nu} .
\end{aligned}
$$

By using Eq. (2) it is easy to show that $W^{\mu}\left(A^{(i)}, A^{(j)}\right)$ is a conserved current: $\nabla_{\mu} W^{\mu}\left(A^{(i)}, A^{(j)}\right)=0$.

A complete set of orthonormal solutions [i.e., complying with Eq. (6)] for Eq. (4) satisfying the constraint (3) can be cast in the form

$$
\begin{aligned}
& A_{\mu}^{\left(\mathrm{I}, \varpi, \mathbf{k}_{\perp}\right)}=C^{\left(\mathrm{I}, \varpi, \mathbf{k}_{\perp}\right)}\left(0,0, k_{y} \phi,-k_{x} \phi\right), \\
& A_{\mu}^{\left(\mathrm{II}, \varpi, \mathbf{k}_{\perp}\right)}=C^{\left(\mathrm{II}, \varpi, \mathbf{k}_{\perp}\right)}\left(\partial_{\xi} \phi,-i \varpi \phi, 0,0\right), \\
& A_{\mu}^{\left(\mathrm{III}, \varpi, \mathbf{k}_{\perp}\right)}=C^{\left(\mathrm{III}, \varpi, \mathbf{k}_{\perp}\right)} \\
& \times\left(-\frac{i \varpi k_{\perp}}{m} \phi, \frac{k_{\perp}}{m} \partial_{\xi} \phi, \frac{i k_{x} \rho^{2}}{m k_{\perp}} \phi, \frac{i k_{y} \rho^{2}}{m k_{\perp}} \phi\right),
\end{aligned}
$$

where

$$
\begin{aligned}
C^{\left(\mathrm{I}, \varpi, \mathbf{k}_{\perp}\right)} & =\sqrt{\sinh (\pi \varpi / a)} /\left(2 \pi^{2} k_{\perp} \sqrt{a}\right), \\
C^{\left(\mathrm{II}, \varpi, \mathbf{k}_{\perp}\right)} & =\sqrt{\sinh (\pi \varpi / a)} /\left(2 \pi^{2} \rho \sqrt{a}\right), \\
C^{\left(\mathrm{III}, \varpi, \mathbf{k}_{\perp}\right)} & =\sqrt{\sinh (\pi \varpi / a)} /\left(2 \pi^{2} \rho \sqrt{a}\right) .
\end{aligned}
$$

Here

$$
\phi\left(x^{\alpha}\right) \equiv K_{i \varpi / a}\left(\rho e^{a \xi} / a\right) e^{i\left(k_{x} x+k_{y} y-\varpi \tau\right)},
$$

where $\rho \equiv \sqrt{k_{\perp}^{2}+m^{2}}, k_{\perp} \equiv\left|\mathbf{k}_{\perp}\right|$, and $K_{i \varpi / a}\left(\rho e^{a \xi} / a\right)$ is the Bessel function of imaginary order [10]. It is interesting to note also that it does exist a forth mode:

$$
A_{\mu}^{\left(\mathrm{IV}, \varpi, \mathbf{k}_{\perp}\right)}=\frac{\sqrt{\sinh (\pi \varpi / a)}}{\left(2 \pi^{2} m \sqrt{a}\right)}\left(-i \varpi \phi, \partial_{\xi} \phi, i k_{x} \phi, i k_{y} \phi\right)
$$

with negative norm,

$$
\left(A^{\left(\mathrm{IV}, \varpi, \mathbf{k}_{\perp}\right)}, A^{\left(\mathrm{IV}, \varpi^{\prime}, \mathbf{k}_{\perp}^{\prime}\right)}\right)=-\delta\left(\varpi-\varpi^{\prime}\right) \delta^{2}\left(\mathbf{k}_{\perp}-\mathbf{k}_{\perp}^{\prime}\right),
$$

orthogonal to all $A_{\mu}^{\left(\lambda, \varpi, \mathbf{k}_{\perp}\right)}(\lambda=\mathrm{I}, \mathrm{II}, \mathrm{III})$. Although it satisfies Eq. (4), it does not comply with the constraint (3) and thus must be considered nonphysical. This reflects the fact that the Proca field has only three independent physical polarizations.

Next, we expand the Proca field in terms of the physical modes as

$$
\begin{aligned}
\hat{A}_{\mu}\left(x^{\nu}\right)= & \int d^{2} \mathbf{k}_{\perp} \int_{0}^{\infty} d \varpi \sum_{\lambda=\mathrm{I}, \mathrm{II}, \mathrm{III}} \\
& {\left[\hat{a}_{\left(\lambda, \varpi, \mathbf{k}_{\perp}\right)} A_{\mu}^{\left(\lambda, \varpi, \mathbf{k}_{\perp}\right)}+\text { H.c. }\right], }
\end{aligned}
$$

where $\int d^{2} \mathbf{k}_{\perp} \equiv \int_{-\infty}^{+\infty} d k_{x} \int_{-\infty}^{+\infty} d k_{y}$. In order to determine the commutation relations between the creation $\hat{a}_{\left(\lambda, \varpi, \mathbf{k}_{\perp}\right)}^{\dagger}$ and annihilation $\hat{a}_{\left(\lambda, \varpi, \mathbf{k}_{\perp}\right)}$ operators, we introduce equal-time canonical commutation relations:

$$
\begin{gathered}
{\left[\hat{A}_{i}(x), \hat{A}_{j}\left(x^{\prime}\right)\right]_{\Sigma}=\left[\hat{\Pi}^{i}(x), \hat{\Pi}^{j}\left(x^{\prime}\right)\right]_{\Sigma}=0} \\
{\left[\hat{A}_{i}(x), \hat{\Pi}^{j}\left(x^{\prime}\right)\right]_{\Sigma}=i \frac{\delta_{i}^{j}}{\sqrt{-g^{(3)}}} \delta\left(\xi-\xi^{\prime}\right) \delta^{2}\left(\mathbf{x}_{\perp}-\mathbf{x}_{\perp}^{\prime}\right)}
\end{gathered}
$$

on the Cauchy surface $\Sigma$ [covered with coordinates $\left(\xi, \mathbf{x}_{\perp}\right)$ with $\left.\mathbf{x}_{\perp} \equiv(x, y)\right]$ among the field operators $\hat{A}_{i}$ and the canonically conjugate momenta $\hat{\Pi}^{j} \equiv n_{\mu} \hat{\pi}^{\mu j}$. Here $i, j$ label space coordinates and Eqs. (17)-(18) are 
cast in a noncovariant form because $\hat{\Pi}^{0}=n_{\mu} \hat{\pi}^{\mu 0}$ vanishes identically and $\hat{A}_{0}$ is not an independent variable (see, e.g., Ref. [11]). Now, on the one hand by using Eq. (16) we obtain

$$
\begin{aligned}
& \left.\left[\left(A^{(i)}, \hat{A}\right),\left(\hat{A}, A^{(j)}\right)\right)\right] \\
& =\sum_{l p}\left(A^{(i)}, A^{(l)}\right)\left[\hat{a}_{(l)}, \hat{a}_{(p)}^{\dagger}\right]\left(A^{(p)}, A^{(j)}\right)
\end{aligned}
$$

(where the sums above should be read as integral symbols for continuous quantum numbers). On the other hand, by using Eqs. (17)- (18), we have

$$
\left.\left[\left(A^{(i)}, \hat{A}\right),\left(\hat{A}, A^{(j)}\right)\right)\right]=\left(A^{(i)}, A^{(j)}\right) .
$$

Thus, by combining Eqs. (19) and (20) and using Eq. (6), we obtain

$$
\left[\hat{a}_{\left(\lambda, \varpi, \mathbf{k}_{\perp}\right)}, \hat{a}_{\left(\lambda^{\prime}, \varpi^{\prime}, \mathbf{k}_{\perp}^{\prime}\right)}^{\dagger}\right]=\delta_{\lambda \lambda^{\prime}} \delta\left(\varpi-\varpi^{\prime}\right) \delta^{2}\left(\mathbf{k}_{\perp}-\mathbf{k}_{\perp}^{\prime}\right)
$$

The Rindler vacuum $|0\rangle_{R}$ is defined by the condition

$$
\hat{a}_{\left(\lambda, \varpi, \mathbf{k}_{\perp}\right)}|0\rangle_{R}=0
$$

\section{THE SOURCE}

Now, let us discuss the source $j^{\mu}(x)$ to which we will couple the Proca field $\hat{A}_{\mu}(x)$ as ruled by the interaction Lagrangian density

$$
\mathcal{L}_{\text {int }}=\sqrt{-g} j^{\mu}(x) \hat{A}_{\mu}(x) .
$$

A uniformly accelerated source in Minkowski spacetime with constant proper acceleration a corresponds to a static source in Rindler coordinates described by the current

$$
j^{\tau}=q \delta(\xi) \delta(x) \delta(y), \quad j^{\xi}=j^{x}=j^{y}=0,
$$

where $q$ will play the role of a coupling constant. According to the Unruh effect, the inertial vacuum corresponds to a thermal state as seen by uniformly accelerated observers confined to the Rindler wedge [4]. Now, because the source is static in Rindler coordinates, it can only interact with zero-energy Rindler particles and, thus, the corresponding spontaneous emission rate must vanish. However, no obvious conclusion can be reached concerning the absorption and induced emission rates because of the divergent number of zero-energy Rindler particles present in the thermal bath. In order to resolve it, we must allow our source to absorb and emit ("respond to", for short) nonzero-energy particles. This can be achieved by introducing a new parameter, $E=$ const, which drives the source to oscillate in time and functions as a regulator. Then, in order to keep charge conservation, we introduce an extra source at $\xi=L$ to form a dipole with our original one. The corresponding conserved current associated with the oscillating dipole, which will temporarily replace our static source (23), can be cast in the form

$$
\begin{aligned}
& j^{\tau}=\sqrt{2} q \cos (E \tau)\left[\delta(\xi)-e^{-2 a L} \delta(\xi-L)\right] \delta(x) \delta(y),(24) \\
& j^{\xi}=\sqrt{2} q E \sin (E \tau) e^{-2 a \xi} \theta(\xi) \theta(L-\xi) \delta(x) \delta(y) \\
& j^{x}=j^{y}=0
\end{aligned}
$$

Eventually, we take the limits $L \rightarrow+\infty$ and $E \rightarrow 0$, and neither the second source at $\xi=L$ nor the charge oscillation will contribute to the final results. We address to Ref. [7] for a more comprehensive discussion on this subject.

\section{EMISSION AND ABSORPTION RATES IN THE UNIFORMLY ACCELERATED FRAME}

In this section, we analyze the total response of our charge with respect to Rindler observers. Using modes (10)-(12) and current (24)-(26) it is obvious that only modes with $\lambda=$ II and III can couple to the charge. We focus first on the emission rate of Rindler particles with $\lambda=$ II.

At the tree level, the emission amplitude of Rindler particles with quantum numbers (II, $\varpi, \mathbf{k}_{\perp}$ ) out to the Rindler vacuum is

$$
\mathcal{A}_{\left(\mathrm{II}, \varpi, \mathbf{k}_{\perp}\right)}^{\mathrm{em}}={ }_{R}\left\langle\mathrm{II}, \varpi, \mathbf{k}_{\perp}\left|i \int d^{4} x \mathcal{L}_{\mathrm{int}}\right| 0\right\rangle_{R} .
$$

The corresponding differential emission rate is given by

$$
d W_{0}^{\mathrm{em}}\left(\mathrm{II}, \varpi, \mathbf{k}_{\perp}\right)=\left|\mathcal{A}_{\left(\mathrm{II}, \varpi, \mathbf{k}_{\perp}\right)}^{\mathrm{em}}\right|^{2} d \varpi d^{2} \mathbf{k}_{\perp} / T,
$$

where $T$ is the (arbitrarily large) proper time interval during which the interaction remains turned on. Taking, at this point, the limit $L \rightarrow+\infty$ to eliminate the influence of the extra source on $d W_{0}^{\mathrm{em}}\left(\mathrm{II}, \varpi, \mathbf{k}_{\perp}\right)$, we obtain

$$
\begin{aligned}
& d W_{0}^{\mathrm{em}}\left(\mathrm{II}, \varpi, \mathbf{k}_{\perp}\right)=\frac{q^{2}}{4 \pi^{3} a} \sinh (\pi E / a) \delta(\varpi-E) \\
& \times\left|K_{i E / a}^{\prime}\left(\frac{\rho}{a}\right)+\mathcal{O}(E)\right|^{2} d \varpi d^{2} \mathbf{k}_{\perp}
\end{aligned}
$$

as $E \rightarrow 0$. (Note that charges at $\xi=L \rightarrow+\infty$ are inertial and, thus, become "inert" for our present purposes.) Now, we should remember that according to the Unruh effect the Minkowski vacuum corresponds to a thermal bath of Rindler particles at a temperature $\beta^{-1}=a /(2 \pi)$. Then, the differential emission rate of massive vector Rindler particles with $\lambda=$ II into the Unruh thermal bath for fixed $\mathbf{k}_{\perp}$ according to uniformly accelerated observers is

$$
P_{\mathrm{II}, \mathbf{k}_{\perp}}^{\mathrm{em}} d^{2} \mathbf{k}_{\perp}=\int d W_{0}^{\mathrm{em}}\left(\mathrm{II}, \varpi, \mathbf{k}_{\perp}\right)\left(1+\frac{1}{e^{2 \pi \varpi / a}-1}\right),
$$

where the first and second terms inside the parenthesis are associated with spontaneous and induced emission, 
respectively, and the integration is performed only in the $\varpi$ variable. By evaluating the integral above and taking the limit $E \rightarrow 0$ at the end, we obtain

$$
P_{\mathrm{II}, \mathbf{k}_{\perp}}^{\mathrm{em}} d^{2} \mathbf{k}_{\perp}=\frac{q^{2}}{8 \pi^{3} a}\left|K_{1}(\rho / a)\right|^{2} d^{2} \mathbf{k}_{\perp} .
$$

The necessity for the introduction of the regularization parameter $E$ can be appreciated from Eq. (28) by noticing that the term that multiplies $\delta(\varpi-E)$ in the right hand side of Eq. (27) vanishes as $\varpi \sim E \rightarrow 0$ while the Planckian (induced emission) term in Eq. (28) diverges as $\varpi \rightarrow 0$. Analogously, we can obtain the differential absorption rate:

$$
P_{\mathrm{II}, \mathbf{k}_{\perp}}^{\mathrm{abs}} d^{2} \mathbf{k}_{\perp}=\int d W_{0}^{\mathrm{abs}}\left(\mathrm{II}, \varpi, \mathbf{k}_{\perp}\right) \frac{1}{e^{2 \pi \varpi / a}-1} .
$$

We see that

$$
P_{\mathrm{II}, \mathbf{k}_{\perp}}^{\mathrm{abs}} d^{2} \mathbf{k}_{\perp}=P_{\mathrm{II}, \mathbf{k}_{\perp}}^{\mathrm{em}} d^{2} \mathbf{k}_{\perp}
$$

because the spontaneous emission becomes negligible in comparison with the induced emission as $E$ vanishes.

Next, we must repeat the same calculations for $\lambda=$ III. It turns out that $P_{\mathrm{III}, \mathbf{k}_{\perp}}^{\mathrm{em}} d^{2} \mathbf{k}_{\perp}=P_{\mathrm{III}, \mathbf{k}_{\perp}}^{\mathrm{abs}} d^{2} \mathbf{k}_{\perp}=0$. This is so because

$$
d W_{0}^{\mathrm{em}}\left(\mathrm{III}, \varpi, \mathbf{k}_{\perp}\right) \sim \mathcal{O}\left(\varpi^{2}\right) \delta(\varpi-E) d \varpi d^{2} \mathbf{k}_{\perp}
$$

as $\varpi \sim E \rightarrow 0$, while $1 /\left(e^{2 \pi \varpi / a}-1\right) \sim \mathcal{O}\left(\varpi^{-1}\right)$ as $\varpi \rightarrow 0$ [and analogously for $d W_{0}^{\text {abs }}\left(\mathrm{III}, \varpi, \mathbf{k}_{\perp}\right)$ ]. As a result, only mode II contributes to the total differential response rate:

$$
\begin{aligned}
P_{\mathbf{k}_{\perp}}^{\text {tot }} d^{2} \mathbf{k}_{\perp} & =\left(P_{\mathrm{II}, \mathbf{k}_{\perp}}^{\mathrm{em}}+P_{\mathrm{II}, \mathbf{k}_{\perp}}^{\mathrm{abs}}\right) d^{2} \mathbf{k}_{\perp} \\
& =\frac{q^{2}}{4 \pi^{3} a}\left|K_{1}(\rho / a)\right|^{2} d^{2} \mathbf{k}_{\perp} .
\end{aligned}
$$

\section{EMISSION RATE IN THE INERTIAL FRAME}

Now, let us compute the response rate for a uniformly accelerated charge as given by Eq. (23) coupled to the Proca field through Eq. (22) with respect to inertial observers. These observers cover the Minkowski spacetime with Cartesian coordinates, in which case the metric components assume the usual form $\eta_{\mu \nu}=$ $\operatorname{diag}(1,-1,-1,-1)$. Hence, it is convenient to write the components of current (23) in the Cartesian basis as

$$
\begin{aligned}
& j^{t}=q a z \delta(x) \delta(y) \delta(\xi), \\
& j^{z}=\operatorname{qat} \delta(x) \delta(y) \delta(\xi), \\
& j^{x}=j^{y}=0,
\end{aligned}
$$

where $\delta(\xi)=\delta\left(z-\sqrt{t^{2}+a^{-2}}\right) /\left(a \sqrt{t^{2}+a^{-2}}\right)$.

An orthonormal set of solutions for Eq. (4) proportional to plane waves and satisfying Eq. (3) can be cast in the form

$$
A_{\mu}(\mathbf{k}, \lambda)=\left(16 \pi^{3} \omega\right)^{-1 / 2} \epsilon_{\mu}(\mathbf{k}, \lambda) e^{i(-\omega t+\mathbf{k} \cdot \mathbf{x})}
$$

with polarization vectors

$$
\begin{aligned}
\epsilon^{\mu}(\mathbf{k}, 1) & =(0, \hat{\epsilon}(\mathbf{k}, 1)), \\
\epsilon^{\mu}(\mathbf{k}, 2) & =(0, \hat{\epsilon}(\mathbf{k}, 2)), \\
\epsilon^{\mu}(\mathbf{k}, 3) & =(|\mathbf{k}| / m, \omega \hat{k} / m)
\end{aligned}
$$

where $\hat{\epsilon}(\mathbf{k}, 1)$ and $\hat{\epsilon}(\mathbf{k}, 2)$ are unit three-vectors orthogonal to each other and to $\mathbf{k} \equiv\left(k_{x}, k_{y}, k_{z}\right), \hat{k} \equiv \mathbf{k} /|\mathbf{k}|$, and $\omega \equiv \sqrt{m^{2}+|\mathbf{k}|^{2}}$. (In this case, the nonphysical mode orthogonal to the physical modes above, satisfying Eq. (4) but not Eq. (3) has polarization $\epsilon^{\mu}(\mathbf{k}, 4)=k^{\mu} / m$.) Next, by using the Klein-Gordon inner product (7) (where in this case the Cauchy surface $\Sigma$ is given by any $t=$ const hypersurface), we verify that the physical modes satisfy

$$
\left(A^{(i)}, A^{(j)}\right)=\delta_{\lambda \lambda^{\prime}} \delta^{3}\left(\mathbf{k}-\mathbf{k}^{\prime}\right),
$$

where $(i),(j)$ represent the quantum numbers $(\mathbf{k}, \lambda)(\lambda=$ $1,2,3)$.

The Proca field in the inertial frame is expanded, thus, as

$$
\hat{A}_{\mu}\left(x^{\nu}\right)=\int d^{3} \mathbf{k} \sum_{\lambda=1}^{3}\left[\hat{a}_{(\mathbf{k}, \lambda)} A_{\mu}(\mathbf{k}, \lambda)+\text { H.c. }\right] .
$$

It is straightforward to verify that $\hat{A}_{\mu}$ and the canonically conjugate momentum $\hat{\Pi}^{\mu}$ satisfy canonical commutation relations provided that (see, e.g., Ref. [11])

$$
\left[\hat{a}_{(\mathbf{k}, \lambda)}, \hat{a}_{\left(\mathbf{k}^{\prime}, \lambda^{\prime}\right)}^{\dagger}\right]=\delta_{\lambda \lambda^{\prime}} \delta^{3}\left(\mathbf{k}-\mathbf{k}^{\prime}\right) .
$$

Now, because inertial observers experience no particles in the Minkowski vacuum $|0\rangle_{M}$ [defined by $\hat{a}_{(\mathbf{k}, \lambda)}|0\rangle_{M} \equiv 0$ for all $(\mathbf{k}, \lambda)$ ], no absorption processes are allowed according to them. As a result, only the emission rate will contribute to the total response. At the tree level, the emission amplitude of a Proca particle with three-momentum $\mathbf{k}$ and polarization $\lambda$ as defined by inertial observers is

$$
{ }^{\mathrm{M}} \mathcal{A}_{(\mathbf{k}, \lambda)}^{\mathrm{em}}={ }_{M}\left\langle\mathbf{k}, \lambda\left|i \int d^{4} x \mathcal{L}_{\mathrm{int}}\right| 0\right\rangle_{M} .
$$

The differential emission rate of Minkowski Proca particles with fixed transverse momentum $\mathbf{k}_{\perp}$ is given by

$$
{ }^{\mathrm{M}} P_{\mathbf{k}_{\perp}}^{\mathrm{tot}} d^{2} \mathbf{k}_{\perp}=\left.\left.d^{2} \mathbf{k}_{\perp} \sum_{\lambda=1}^{3} \int_{-\infty}^{+\infty} d k_{z}\right|^{\mathrm{M}} \mathcal{A}_{(\mathbf{k}, \lambda)}^{\mathrm{em}}\right|^{2} / T .
$$

Next, we use the identity

$$
\sum_{\lambda=1}^{3} \epsilon_{\mu}(\mathbf{k}, \lambda) \epsilon_{\nu}(\mathbf{k}, \lambda)=k_{\mu} k_{\nu} / m^{2}-\eta_{\mu \nu}
$$

satisfied by the polarization vectors to cast Eq. (42) in the form

$$
\begin{aligned}
& { }^{\mathrm{M}} P_{\mathbf{k}_{\perp}}^{\mathrm{tot}} d^{2} \mathbf{k}_{\perp}=d^{2} \mathbf{k}_{\perp} \int_{-\infty}^{+\infty} d k_{z} \int \frac{d^{4} x d^{4} x^{\prime}}{16 \pi^{3} \omega} \\
& \times j^{\mu}(x) j^{\nu}\left(x^{\prime}\right)\left(\frac{k_{\mu} k_{\nu}}{m^{2}}-\eta_{\mu \nu}\right) e^{i \omega\left(t-t^{\prime}\right)} e^{-i \mathbf{k} \cdot\left(\mathbf{x}-\mathbf{x}^{\prime}\right)} .
\end{aligned}
$$


Now, by using current conservation $\partial_{\mu} j^{\mu}=0$, we can show that terms in the integral proportional to $j^{\mu} k_{\mu}$ do not contribute. Finally, by using Eqs. (33)-(35) we find

$$
{ }^{\mathrm{M}} P_{\mathbf{k}_{\perp}}^{\mathrm{tot}} d^{2} \mathbf{k}_{\perp}=\frac{q^{2}}{4 \pi^{3} a}\left|K_{1}(\rho / a)\right|^{2} d^{2} \mathbf{k}_{\perp} .
$$

The equality between Eqs. (43) and (32) can be interpreted in terms of known elementary particles as follows. Firstly, let us note that the Lagrangian density (22) can be used to describe the interaction of noninertial electrons emitting $Z^{0}$ bosons. Then, the equality between Eqs. (32) and (43) reflects, in this case, the fact that each (nonzero energy) $Z^{0}$ emitted from a uniformly accelerated $e^{-}$in the Minkowski vacuum as described by inertial observers corresponds to either the emission to or absorption from the Unruh thermal bath of a zeroenergy $Z^{0}$ Rindler boson as described by coaccelerated observers.

\section{CONCLUDING REMARKS}

We have canonically quantized the Proca field in the Rindler wedge. The results obtained were applied to compute the total response rate of a source with constant proper acceleration interacting with the Proca field from the point of view of observers coaccelerated with the source. We have shown that this corresponds to the combined emission and absorption rates of zero-energy Proca particles to and from the Unruh thermal bath, re- spectively, and that only Rindler particles with $\lambda=$ II contribute. Then, we have explicitly verified that the total response rate obtained in the Rindler wedge is exactly the same as the total emission rate of Minkowski particles as described by inertial observers. This result is consistent with the fact that each particle emitted in the inertial frame must correspond to either the emission or absorption of a Rindler particle in the accelerated frame, since both observers must agree concerning changes in the state of the quantum field. Finally, because of the close conceptual relationship between the Unruh thermal bath and Hawking radiation, we can forecast that the total response of a static source in the vicinity of a black hole coupled to the Proca field will be well approximated by $\int P_{\mathbf{k}_{\perp}}^{\text {tot }} d^{2} \mathbf{k}_{\perp}$, where $P_{\mathbf{k}_{\perp}}^{\text {tot }} d^{2} \mathbf{k}_{\perp}$ is given in Eq. (32) with $a$ being identified with the proper acceleration of the static source outside the black hole.

\section{Acknowledgments}

The authors are grateful to Conselho Nacional de Desenvolvimento Científico e Tecnológico and to Coordenação de Aperfeiçoamento de Pessoal de Nível Superior for partial financial support. J.C., E.C., and L.C also acknowledge Fundação de Amparo à Pesquisa do Estado do Pará while G.M. is grateful to Fundação de Amparo à Pesquisa do Estado de São Paulo for partial support. We are indebted to Atsushi Higuchi for various discussions.
[1] W. G. Unruh, Phys. Rev. D 14, 870 (1976).

[2] N. D. Birrell and P. C. W. Davies, Quantum Field Theory in Curved Spacetime (Cambridge University Press, Cambridge, 1982).

[3] S. A. Fulling and S. N. M. Ruijsenaars, Phys. Rep. 152, 135 (1987).

[4] L. C. B. Crispino, A. Higuchi, and G. E. A. Matsas, Rev. Mod. Phys. 80, 787 (2008).

[5] H. Ren and E. J. Weinberg, Phys. Rev. D 49, 6526 (1994).

[6] J. Castiñeiras, I. P. Costa e Silva, and G. E. A. Matsas, Phys. Rev. D 67, 067502 (2003).
[7] A. Higuchi, G. E. A. Matsas, and D. Sudarsky, Phys. Rev. D 46, R3450 (1992).

[8] G. E. A. Matsas and D. A. T. Vanzella, Phys. Rev. D 59, 094004 (1999).

[9] J. Castiñeiras, L. C. B. Crispino, G. E. A. Matsas, and D. A. T. Vanzella, Phys. Rev. D 65, 104019 (2002).

[10] I. S. Gradshteyn and I. M. Ryzhik, Tables of Integrals, Series, and Products (Academic Press, New York, 1980).

[11] W. Greiner and J. Reinhardt, Field Quantization (Springer, Berlin, 1996). 